\title{
APLICACIONES DE TECNOLOGÍA LINGÜISTICA Y OTROS RECURSOS DIDÁCTICOS PARA LA ENSEÑANZAAAPRENDIZAJE DEL ESPAÑOL COMO LENGUA EXTRANJERA: EL VERBO
}

\author{
Herminia Provencio Garrtgós \\ Universidad de Alicante. Grupo de Investigación Griale \\ Herminia.Provencio@ua.es
}

\begin{abstract}
Resumen
In this article, we pretend to show a series of softwarc applications on Linguistic Technology (ATL is the acroym in Spanish) and others Spanish-like-foreign-language (ELE) tcaching/learning resources on-line (Internet resources). Our classification has four kinds of resourecs: a) writing helpers, b) verbal conjugers, c) verbal gramatic information webpages, and d) verbal practice exerciscs. We identify the contexts of the ELE classroom development where it's suitable to use these resourccs. At last, we offer a scholars template to evaluate this resources usage.
\end{abstract}

\section{La Tecnología Lingúística}

En la actualidad lo tecnológico, lo deseemos o no, está entrando en nuestra intcractividad diaria y, por supuesto, en nuestra labor docente: interactuamos con alumnos, con profesores y con materiales/recursos, estos últimos accesibles en muchos casos por medio de aplicaciones tecnológicas.

Al hablar de Aplicaciones de Tecnología Lingüística (ATL), nos referimos, siguiendo a $\mathrm{M}^{\mathrm{a}}$ A. Marti Antonín y a J. Llisterri, a aquellas que intentan «reconocer, comprender, interpretar y generar el lenguaje humano en todas sus formas» (2001:3). Tradicionalmente se distingue entre: tecnologias del texto escrito y tecnologías del habla, en este lugar se abordarán sólo algunas aplicaciones de las primeras ${ }^{1}$. Ambas tecnologías son el resultado de la unión entre el conocimiento lingüístico y el informático al que habrá que sumarle el uso de Internet, como medio que nos va a proporcionar la interacción comunicativa con esas aplicaciones; por este motivo, nuestro interés se va a centrar en los que están disponibles en línea, ya que partimos de la premisa de que Internet constituye en la actualidad una de las mayores fuentes de rccursos para la enseñanza/aprendizaje del español como lengua extranjera (E/LE).

A lo largo de estas páginas intentaremos demostrar que estas aplicaciones/recursos, como constatan Arrarte y Llisterri (1997:31 y ss), pueden favorecer, si se orientan oportunamente, la enseñanza/aprendizaje de nuestra lengua, y podemos servirnos de ellas para la práctica

1 Para las tecnologías del habla consúltese la excclentc y completa página personal de J. Llisterri: http:/liceu. uab.es/-joaquim/ 
docente y concreta de determinadas cucstiones verbales, pero sin olvidar que «los medios son para la didáctica y no la didáctica para los medios» (Fernández Pinto, 2003-04).

\section{EL VERBO EN LA RED: ATL Y OTROS RECURSOS PARA SU APRENDIZAJE}

Establecer una taxonomía específica sobre las aplicaciones y recursos que hay en la red para integrarlos en el proceso de enseñanza/aprendizaje de cuestiones verbales es una labor relativamente scncilla, lo que resulta complejo es ir complementando esa taxonomía (que hemos estructurado en los epígrafes siguientes) con sitios web; sería un despropósito por nuestra parte si así fuera, ya que todos somos conscientes de lat volatilidad de muchos de ellos. Los que hemos seleccionado creemos que responderán a los objetivos que nos hemos marcado en los primeros párrafos de este artículo.

\subsection{Aplicaciones de ayuda a la escritura (redacción y corrección)}

Estas aplicaciones sirven para realizar la corrección ortográfica, gramatical y de cstilo de un texto $^{2}$. Se integran en los procesadores de texto y son conocidas también bajo el nombre de herramientas (Llisterri, 2003b:19). Aunque estas aplicaciones no están en línea podemos decir que son la primera ayuda, quc sin pedirla, se le ofrece al estudiante y el feedback es inmediato, sobre todo, como comenta L. Casanova, cuando aparece el denominado fenómeno de fosilización consistente en la repetición de los mismos errores de forma reiterativa durante un periodo de tiempo los correctores pueden ser de gran ayuda al alumno (1998:20).

Fs ya muy frecuente que los estudiantes utilicen el ordenador como herramienta para su expresión c interacción escrita, lo que favorece el desarrollo de «estrategias de escritura» (Arrarte y Llisterri, 1997:31). Así, por ejemplo, podrá ver si la forma verbal que ha utilizado es gramaticalmente corrccta o no; pero también debe de saber, como usuario de esta herramienta en otras lenguas, que no siempre son fiables sus correcciones, aunque todos sabemos que los avances en cste terreno han sido y serán muchos, solo hay que recordar los procesadores que ulilizábamos hace unos años y los actuales, que incorporan prestaciones cada vez más perfectas. Pero esto también tienc su lado contrario, el alumno se relaja en el proceso de escritura y deja de prestar atención a determinados aspectos de su ortografía, gramática o estilo.

Dentro de las aplicaciones de ayuda a la eseritura se encuentran también aquellas desarrolladas por empresas privadas dedicadas al desarrollo de software do tecnología lingüística que pueden integrarsc cn los procesadores de texto o estar accesibles en Internet para cualquier consulta. En este apartado, hemos seleccionado dos sitios web:

a) Signum. Lenguaje.com. El sitio de la ingenieria del lenguaje (http://lenguaje.com). Ofrece diez productos o herramientas para facilitar el trabajo de escritura; el específico para la corrección ortográfica es el que dcnominan: verificador ortográfico (corrector de ortografía para el español: htlp://www.lenguaje.com/herramientas/verificador/default.htm). El alumno puede escribir una palabra o una frase y a continuación se le mostrarán las sugerencias de corrección.

2 Entendemos por texto, siguiendo a Vera Luján: «la menor unidad dotada de autonomía comunicativu» (1994:31), por lo tanto, un texto podrá ser una novela completa o un enunciado como: ¡ven! 
b) DAÉDALUS. Data, Decisions and Language, S. A. (http://www.daedalus. es/PerfilE.php). Empresa que ofrece como principal producto dentro de su área de producción en Tecnología Lingüística: STILUS corrector ortográfico, gramatical y de estilo (http://www. daedalus.es/demo/stilus/) (imagen 1), que permite revisar cualquier texto escrito en español, ya se trate de un documento generado con nuestro procesador de textos (esta opción puede resultar muy provechosa para el alumno al no tener que teclear sus propios escritos), de una página web o de un texto que se escriba en el formulario de la página de Stilus; y obtener un informe detallado y preciso con los errores cometidos, aunque con las oportunas reservas sobre determinadas correcciones.

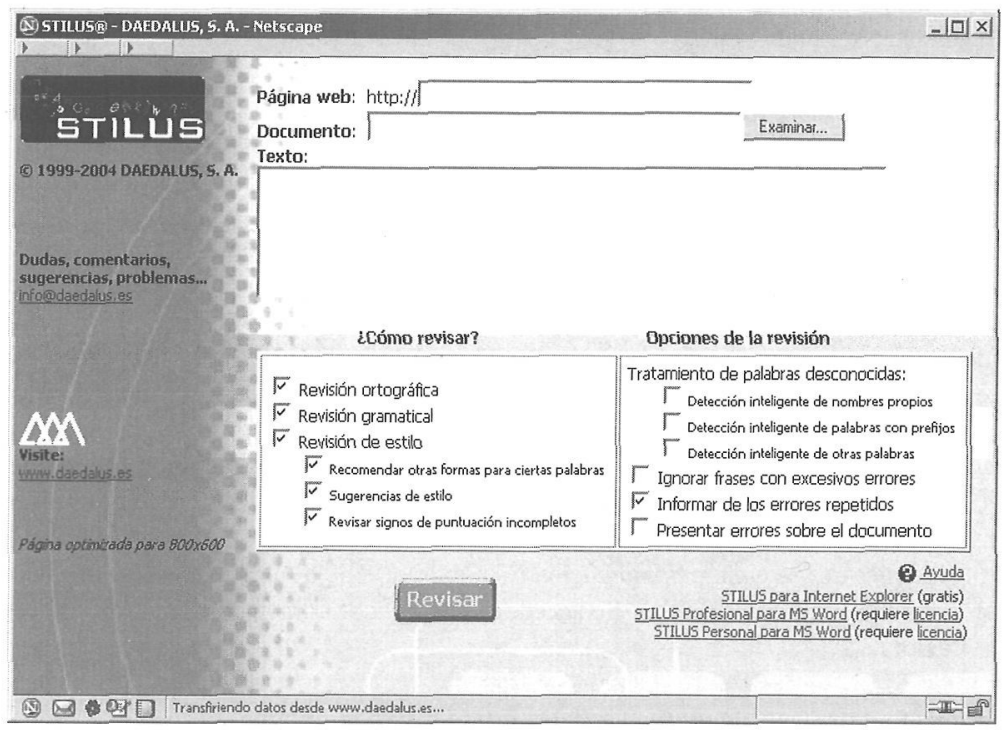

Imagen 1: STILUS corrector ortográfico, gramatical y de estilo

\subsection{Conjugadores/flexionadores verbales interactivos simples}

Tal y como indica el epígrafe, son los conocidos recursos en línea que permiten introducir un infinitivo verbal (lema) y a partir de ahí se flexiona todo el verbo ${ }^{3}$. Consideramos a estos conjugadores simples ya que la respuesta que ofrecen es la conjugación completa. Para este lugar hemos seleccionado ocho:

3 Sería interesante, si se les van a facilitar a los alumnos las direcciones web para realizar la conjugación, proporcionarles una equivalencia terminológica sobre la variabilidad de formas de nombrar algunos tiempos verbales, por ejemplo: pretérito perfecto compuesto $=$ pretérito perfecto $=$ antepresente; pretérito perfecto simple $=$ pretérito indefinido = pretérito; pretérito imperfecto de indicativo = copretérito, etc. 
a) Conjugador de verbos del Grupo de Sintaxe do Español de la Universidad de Santiago (http://www.sintx.usc.es/Frames.html).

b) Verbix. Verb Conjugations Grammar (http:/www.verbix.com/languages/ spanish. shtml). La lengua vehicular de esta página es el inglés y da los infinitivos equivalentes en otras lenguas.

c) Conjugador de la Real Academia Española (RAE) (http://buscon.rac.cs/ diccionario/drac.htm) Para obtener la conjugación de un verbo hay que acceder al diccionario, escribirlo y cuando aparezca las acepciones, pulsar sobre el botón en azul que aparece delante del lema verbal.

d) Comp-jugador creado por D. M. Germán de la Universidad de Waterloo (http://turingmachine.org/compjugador/)

e) Conjugador de verbos del Servicio Común de Informática Gráfica de la Universidad de Oviedo (http://tradu.scig.uniovi.es/conjuga.html)

f) Conjugador (herramienta de conjugación verbal) de la empresa Signum. Lenguaje. com. El sitio de la ingeniería del lenguaje (http:/www.lcnguaje.com/ herramientas/conjugador/default.htm

g) Instituto de Verbología Hispánica (http://www.verbolog.com). Este sitio tiene diversos cnlaces con distintas funcionalidades, el acceso al conjugador es: el enlace hipertextual Inventario General y Conjugador Avanzado de 90.970 verbos distintos del español y sus dialectos (http://www.verbolog.com/igcaved.htm). Al escribir el infinitivo aparece una ventana con distintos tipos de información sobre el verbo y pinchando sobre Modelo de FLEXIÓN nos saldrán todas sus formas flexionadas. También podemos buscar a través de su enlace Los 100 modelos de conjugación en español (http://www.verbolog.com/conjuga. $\underline{\mathrm{htm}}$ ), que, como su propio nombre indica, muestra la conjugación de cien verbos pulsando sobre sus infinitivos.

h) Centro de lenguaje y Computación (CliC) (http://clic.fil.ub.es/) empresa que depende de la Universidad de Barcelona. Se accede al conjugador a través de: Demos $>$ morfología > flexionador.

\subsection{Flexionadores/conjugadores verbales interactivos complejos}

La diferencia de estos flexionadores con respecto a los anteriores es que permiten realizar la flexión sólo de aquellos tiempos y formas verbales que el usuario precise, proporcionando algunos de ellos otras informaciones, por ejemplo, de carácter semántico.

Estas herramientas pueden ser do dos tipos:

a) Herramientas que hacen la flexión a partir del lema verbal (infinitivo):

La sede web que hemos escogido es el Flexionador de la empresa Signum. Lenguaje.com. El sitio de la ingenieria del lenguaje (http:/www.lenguaje.com/ herramientas/flexionador/default.htm). Desde esta aplicación se puede scleccionar a partir de un infinitivo la flexión que se desee en cuanto al modo, tiempo, persona, enclíticos, etc. Los resultados que ofrece son para la persona verbal que hayamos seleccionado. En la imagen 2 se visualiza la búsqueda de la $1^{\text {a }}$ pcrsona del plural del futuro de subjuntivo, y en la misma ventana se ofrece el resultado: amóremos.

b) Herramientas que hacen la flexión a partir de cualquier forma verbal. El prototipo de esta herramienta lo encontramos en las ATL desarrolladas en el Grupo de Estructura de 


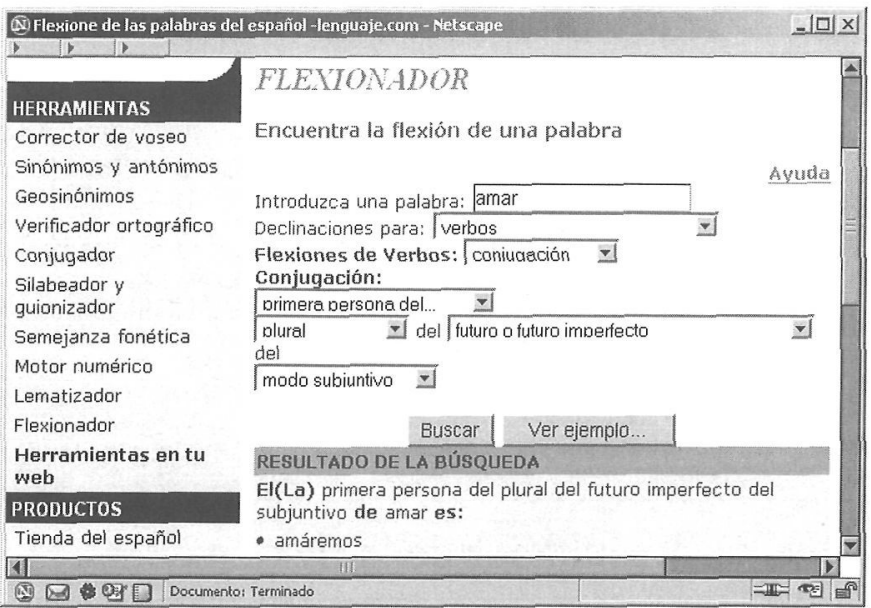

Imagen 2: Flexionador de Signum.

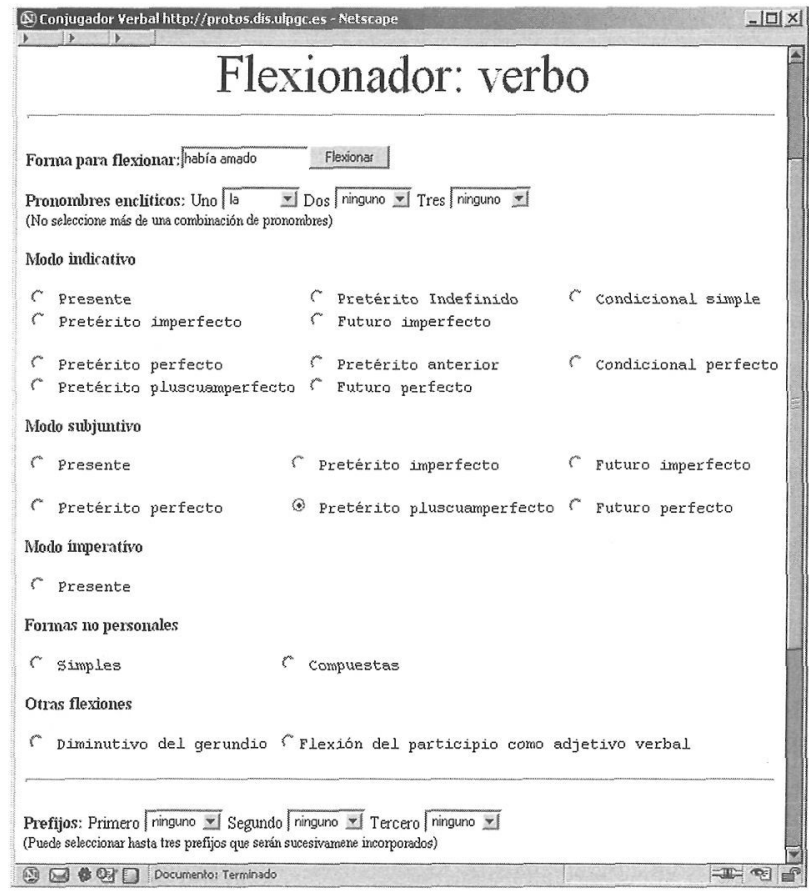

Imagen 3: FLAVER Flexionador del GEDLC - I 
Datos y Lingüística Computacional (GEDLC) (Universidad de Las Palmas de Gran Canaria) y, en concreto, en la aplicación informática FLAVER: conjugador/flexionador verbal (http://gedlc.ulpgc.es/ investigacion/scogeme02/flexver.htm) en la que el usuario introduce cualquier forma univerbal o pluriverbal — en nuestro caso habia amado (imagen 3) - y se le proporciona su forma canónica (lema), categoría e información sobre su flexión (descripción morfológica: tiempo, modo, persona y número -imagen 4-). Desde la misma ventana (imagen 3) es posible solicitar la flexión/conjugación de esa forma en otro tiempo/modo y con la opción de seleccionar el uso de pronombres enclíticos y de prefijos. En la imagen 3 hemos seleccionado: pronombre enclítico la, modo subjuntivo: pretérito pluscuamperfecto, prefijo: ninguno y la retroalimentación sería la imagen 4.

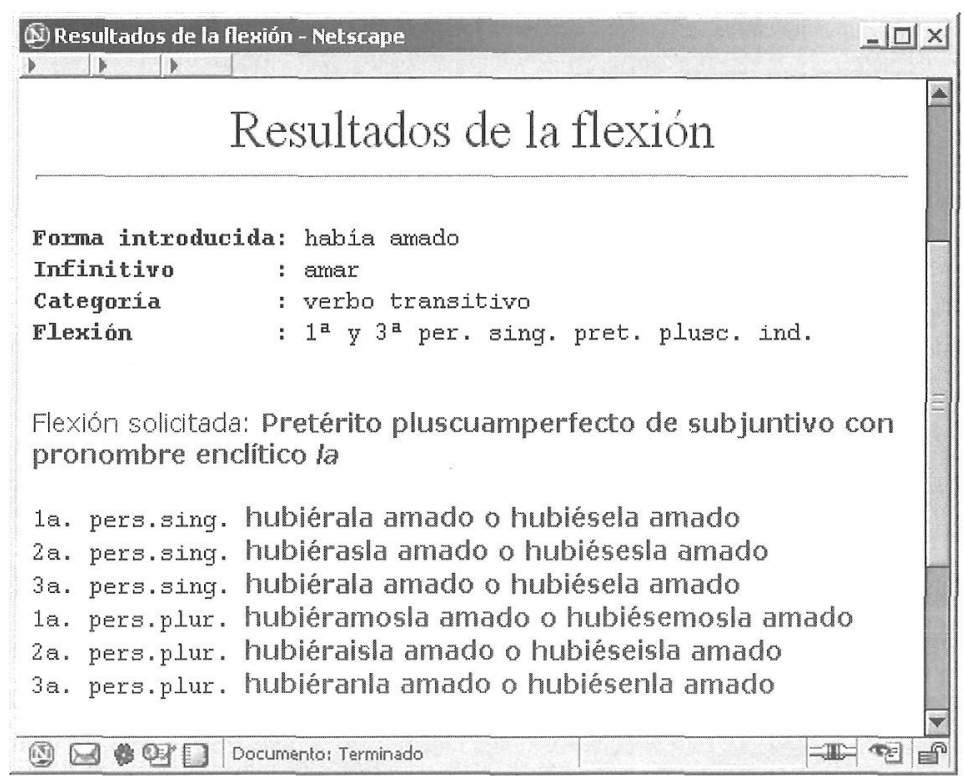

Imagen 4: FLAVER Flexionador del GEDLC - II

Otra forma de acceder a la información sobre la flexión de una forma verbal es a través de los lematizadores verbales. El Grupo de Estructura de Datos nos proporciona la aplicación FLAPE: flexionador y lematizador de palabras en español (http://www.gedlc.ulpgc. es/investigacion/scogeme02/lematiza.htm). Los resultados que ofrece al introducir una forma verbal son los mismos que en el conjugador/flexionador verbal (imagen 5), aunque se añade una clasificación semántica y dos hipervínculos: uno hacia el flexionador (imagen 3) y otro hacia relaciones morfoléxicas (derivación, palabras relacionadas y regularidad de la forma solicitada). 


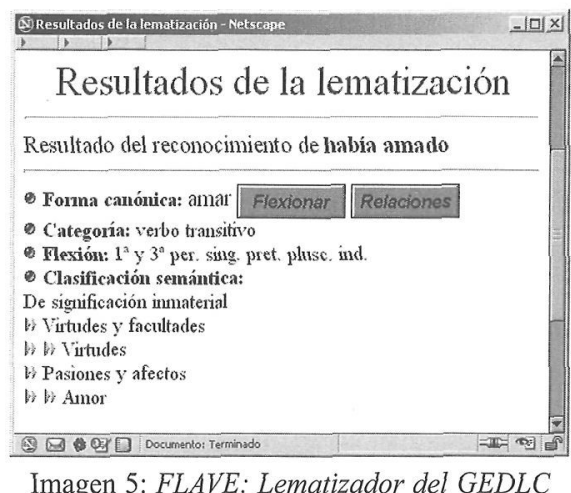

El Centro de lenguaje y Computación (CliC) (http://clic.fil.ub.es/), empresa que depende de la Universidad de Barcelona, tiene también disponibles en red diferentes ATL que proporcionan información morfológica (flexionador, lematizador y etiquetador) y sintáctica sobre las formas verbales. Por ejemplo, en la imagen 6 se le pide a la aplicación que lematice amaba y la retroalimentación ofrece el lema más la descripción morfológica de la forma.

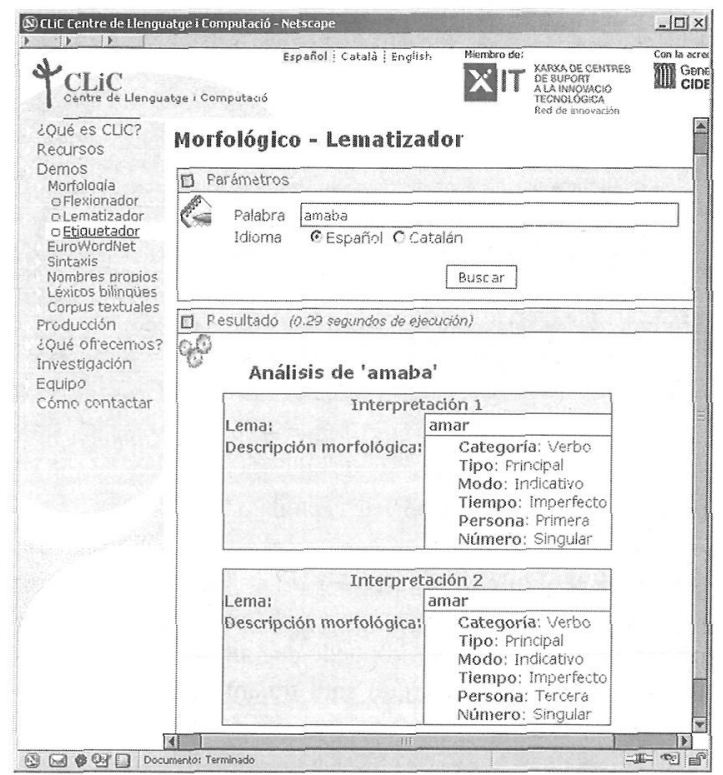

Imagen 6: Analizador morfológico de $\mathrm{CliC}$ 
Hemos seleccionado dentro de este grupo el conjugador que nos ofrece el sitio Logos Group: Verba Universal Conjugador (http://www.verba.org). Se introduce una forma verbal y se conjuga todo el verbo, aunque a partir de algunas formas compuestas no da acceso a la conjugación completa y nos pide que introduzcamos otra forma. Presenta un rasgo que lo diferencia de los visto hasta ahora y es el hecho de que es posible acceder a varios contextos en los que aparece cada forma verbal, pinchando en cada una de ellas (imagen 7).

\begin{tabular}{|c|c|}
\hline \multicolumn{2}{|l|}{ 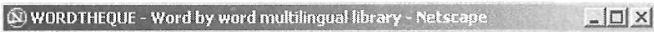 } \\
\hline \multicolumn{2}{|l|}{ 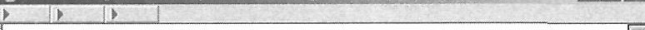 } \\
\hline \multicolumn{2}{|l|}{$\begin{array}{l}\text { Context information for: he amado } \\
\text { Match N. } 1 \text { Error in procedure w6_context.display_context } \\
\text { ORA-01403: no data found } \\
\text { Match N. } 2\end{array}$} \\
\hline \multicolumn{2}{|l|}{$\begin{array}{l}\text { Author: Garmona Ernesto (Editor) } \\
\text { Title: MORIR ES LA NOTICIA } \\
\text { Source: http://shop.logos.it/users/carmona/index } \\
\text { Subject: THE POLITICAL PROCESS (324) }\end{array}$} \\
\hline $\begin{array}{l}\text {... Algún dia, tal vez,/ en cualquier calle/ o en alguna celda,/ } \\
\text { con mi espalda ardiendo/ contra una muralla fría,/ yo muera.../ } \\
\text { Mi pueblo te agradecera / el amor que has hecho renacer en } \\
\text { mí... / Sé feliz y recuérdame,/ en silencio te he amado/ y mi } \\
\text { último grito que sea de triunfo y de amor". Guillermo Montecinos, } \\
\text { fallecido en } 1990 \text { por las secuelas de un balazo sufrido en años } \\
\text { anteriores, recopiló todos sus escritos y los encabezó así: "Son } \\
\text { parte de los poemas }\end{array}$ & i \\
\hline \multicolumn{2}{|l|}{ Match N. 3} \\
\hline $\begin{array}{l}\text { Author: Valera Juan } \\
\text { Title: EL CAUTIVO DE DOÑA MENCÍA } \\
\text { Source: ht tp://www.cervantesvirtual.com/servlet/Sirveobras } \\
\text { Subject: SPANISH MISCELLANEOUS WRITINGS (868) }\end{array}$ & \\
\hline $\begin{array}{l}\text {... qué profundo convencimiento, qué fe y qué segura esperanza } \\
\text { en tus futuros y soberanos destinos. Sí, yo no he amado sólo en } \\
\text { tu persona al gallardo y floreciente mancebo en toda la frescura } \\
\text { y lozanía de su edad primera. Yo he amado y prefigurado en ti al } \\
\text { héroe en flor, gloria y grandeza de la patria, al que contribuirá } \\
\text { más que nadie a que castilla, disuelta hoy en bandos y asolada } \\
\text { por guerras civiles, con España toda unida a Castilla, sea }\end{array}$ & $\frac{1}{1}$ \\
\hline 11 1 तil & $\mid$ \\
\hline (9) & \\
\hline
\end{tabular}

Imagen 7: Contextos verbales del Verba Universal Conjugador

\subsection{Sitios con información sobre gramática verbal}

En este epígrafe y en el siguiente (Ejercicios de práctica verbal), propondremos sólo una breve lista de materiales disponibles en red en las que se puede encontrar información gramatical, ya que el lector de este artículo puede encontrar en múltiples sedes web información actualizada sobre las cuestiones que aquí tratamos; destacamos, por ejemplo, los siguientes:

$4 \quad$ La lengua vehicular es el inglés. 
a) La web de la Asociación para la Enseñanza del Español como Lengua Extranjera (ASELE) (http:/www.aselered.org) y en su enlace hipertextual «Enlaccs» expone una relación de recursos gratuitos disponibles en Internet útiles para la enseñanza de ELE $^{5}$.

b) Recomendamos acceder al metarrecurso Lingu@net Europa (Centro de Recursos Virtual y multilingüe para la enseñanza de lenguas) (http://www. linguanet-europa.org/) y a su enlace Materiales para la enseñanza (Arrarte y Sánchez de Villapadierna, 2002).

c) Muchas de las revistas especializadas tienen secciones dedicadas a las TIC, por ejemplo, Cuadernos Cervantes de la lengua española. La Revista del Español en el mundo (http://www.cuadernoscervantes.com/multimedia.htm); la revista Carabela en su sección Recursos en Internet para la elaboración de actividades (sección incluida en 1998) accesible también desde el enlace Contexto culturales hispánicos en los Medios de Comunicación y en las Nuevas Tecnologias (http://www.ub.es/filhis/culturele/nue_tecn.html - http://www. ub.es/filhis/culturele/carabela.html) de la revista Cultura e Intercultura en la enseñanza de ELE ( http://www.ub.es/filhis/culturele/00.htm); y, Mosaico. Revista para la promoción y apoyo a la enseñanza del español: El español en Internet (http://www.sgci.mec.es/be/publicaciones/mosaico/normas.htm).

d) La base de datos que elaboró M. Cruz Piñol (2002) en la que analizaba pormenorizadamente 207 web útiles para la enseñanza de $\mathrm{E} / \mathrm{LE}$, accesible desde la url: http://www. octaedro.com/ele/fitxab.asp.

A continuación, referimos una muestra de sitios web, algunos ya clásicos, en los que poder encontrar material informativo sobre gramática verbal:

a) Gramática y ortografia. Página de Juan Manuel Soto Arriví. Reglas gramaticales (http://www.indiana.edu/ call/reglas.html).

b) El verbo en nuestra lengua. Página de Ricardo Soca. El castellano.org. La página del idioma español. (http://www.elcastellano.org/esbverb.html).

c) Guía de gramática y Libro de gramática. Página de Enrique Yepes. Bowdoin College Department of Romance Languages (http://www.bowdoin.edu/ cyepes/newgr/gramguid. htm, http://www.bowdoin.edu/ eyepes/newgr/ats/index .htm).

d) El verbo. Página de Sergio Zamora. La Nueva Lengua Española. El verbo. http:// www.geocities.com/sergiozamorasin/elverbo.htm

e) Zona Ele. Recursos para alumnos y profesores de español como lengua extranjera. Material on-line. Gramática española / Estudia gramática/ ¿Los verbos son dificiles? (http://www.zonaele.com/home.htm)

f) Lengua y Literatura. Educación Secundaria. Página de Mariano Santos Posada. (http://roble.pntic.mec.es/ msantol/lengua/) Ofrece contenidos seguidos de actividades con dos niveles: repaso y profundización.

g) Morfologia española en tablas. El verbo. Rincón de la lengua española. (http://www. iespana.es/morfologia es/morfologia/)

h) Instituto Cervantes. Recursos para la preparación de la prueba de expresión escrita (D.E.L.E). Ayuda de gramática. Nivel Inicial (http:/internet. cervantes.es/internetcentros/ ensenanza/materiales/dele/marcos_inicial/frameset_inicial.html).

5 La información está actualizada y la página está mantenida por Mar Cruz Piñol. Para los objetivos de cstc artículo destacimos las secciones: Páginas sobre morfología y sintaxis, Páginas sobre nownativa, ortografía y dudas, Páginas con ejercicios estructurales tipo "test" y ejercicios lúdicos de corrección automática y Páginas con ejencicios sobre la conjugación verbal y la formación de palabras 
i) El juego. Fichas de gramática. El verbo. (http://www.eljuego.net/)

\subsection{Ejercicios de práctica verbal ${ }^{6}$}

Cuando hablamos de ejercicios/actividades en red, debemos pensar que, en principio, existe una diferencia básica con respecto a los de formato clásico o papel. Si no fuera así estaríamos anulando las posibilidades que nos brinda las TIC (Tecnologias de la Información y la Comunicación). ¿Cuál es la potencialidad de las actividades en red?, evidentemente, la intcractividad, el hipertexto y el multimedia.

La mayoría de los ejercicios en línea presentan bastantes similitudes con los que están en formato papel; pero a pesar de ello podemos encontrar algunas ventajas para realizarlos en este medio, Cruz. Moya menciona las siguientes: la corrección del ejercicio es inmediata, disminuye la ansiedad del alumno, cl alumno se suele sentir más motivado que con las mismas actividades en soporte papel, el alumno puede elegir el ejercicio que le es más adecuado a su nivel aprendizajc (2002:542), con la práctica de estos ejercicios el alumno puede mejorar su ritmo de aprendizaje, etc.

No obstante, los inconvenientes también cxisten, y podemos decir que en la actualidad la mayoría de los ejercicios disponibles en red que tratan sobre cuestiones verbales son de respuesta cerrada, no suelen ofrecer ayudas y la corrección es automática. Una característica que, de momento, comparten muchos de los ejercicios en red, traten aspectos léxicos, gramaticales, culturales, etc. es lo que se ha venido en llamar ciberestructuralismo, es decir una vuelta hacia el enfoque estructural: rellenar huecos, asociaciones, elección múltiple, reconocimiento, etc., que poco se diferencian de los existentes en la enorme oferta de manuales que hay en el mercado (Martín Mohedano, 2003:41; Cruz Piñol, 2002:107-108, 133-136,158, 142-143, 2004, Arrarte y Sánchez de Villapadierna, 2001:56-61, Baralo Ottonello, 2002:493-494). El problema radica en saber aprovechar el polencial didáctico que las TIC nos brindan, un potencial que muchas veces se ve eclipsado por todos los puntos de amarre en los que tienc que sustentarse: preparación del profesor para integrar las TIC en el contexto del aula y su dedicación a las tutorías presenciales más las virtuales ${ }^{7}$, recursos informáticos disponibles èn el centro y en el aula, etc. (Gallego Arrufat, 1998, Arrarte y Sánchez de Villapadierna 2001:62-73; Juan Lázaro, 2001: 13-20).

Para los ejercicios seleccionados, que son una mucstra representativa de los que actualmente están colgados en red, hablariamos de ejercicios interactivos de práctica individual, aunque no hay que descartar su utilidad para trabajarlos en parcjas en una clase presencial:

a) Página de Juan Manuel Soto Arriví. Ejercicios en la red. Gramática. http://www. indiana.edu/ call/ejerci.html

b) Página de Juan Ramón Arana. Modern Language Departament Ursinus College. Spanish Language Exercises (http://mld.ursinus.edu/ jarana/Ejercicios/instructors/verbs.html

6 Podemos encontrar un análisis y valoración de algunas de las páginas que mencionamos en este apartado cn el trabajo de Grande Rodriguez y Grande Alija (2002:544-548).

7 Considero que Cruz Piñol (2004) señala de forma muy esclarecedora uno de los problemas con los que se está tropezando la enseñanza presencial y el uso, en este caso, de Intemet: las horas exiras que los docentes deben dedicar a las futorias telemáticas, aparte las tutorías presenciales. 
c) Página de Barbara Kuczun Nelson. Spanish Grammar Exercices (hltp:/www.colby. edu/\%7Ebknelson/exercises/

d) Hispanic Studies @, Trentu University. Ejercicios que complementan cl manual /Arriba! Comunicación y Cultura (http://www.trentu.ca/spanish/ masarriba/)

e) Ejercicios interactivos de Aula Diez. Español on-line (http://www, auladiez.com/ejercicios/index.html). Para acceder a todos los ejercicios hay que matricularse en los cursos, pero es posible acceder de forma gratuita a algunos de ellos.

f) Quia http://www.quia.com/dir/spanish/

g) Comunicativo.net. International House Madrid S.A. Interactivo. Juegos y actividades para aprender español. (http://www.studyspanish,com/tutorial.htm). Los ejercicios interactivos que ofrece son para trabajar los tiempos en pasado.

h) Página de Enrique Yepes. Bowdoin College Department of Romance Languages. Ejercicios de gramática en la red digital. (http:/www.bowdoin.edu/ cyepes/gramex.htm). Ofrece una relación clasificada de ejercicios interactivos en la que incluye los que él ha realizado.

i) Gram@clicando - Ejercicios de granática española. El verbo. http://pot-pourri.fltr. ucl.ac.be/gra/

Queremos mencionar también, aunque es un lugar web conocido por todos, el Centro Virtual Cervantes (http://cvc.cervantes.es/portada.htm), sitio al que se recurre por la calidad de sus materiales y actividades. En su sección Aula de lengua (http:/cvc.cervantes.es/aula/) y dentro de ella en Ios recursos: Diplomas de Español (http://cvc.cervantes.es/aula/dele/), Pasatiempos de Rayuela (http:/cvc.ccrvantes.es/aula/pasatiempos/) y Lecturas paso a paso (http://cvc.cervantes.es/aula/lecturas/), el profesor encontrará actividades para que el alumno trabaje de forma autónoma, en parejas o en pequeños grupos. En Pasatiempos se pueden seleccionar actividades directamente por contenido gramatical y por nivel. El tipo de interacción que predomina para las de contenido gramatical son: sopas de letras, crucigramas y opción múltiple. Destacamos para este lugar las siguientes actividades que versan sobre el uso de los tiempos verbales en pasado:

a) Historias de la Cava (http:/cvc.cervantes.es/aula/pasatiempos/ pasatiempos2/ avanzado/gramatical/03082000_01.htm)

b) Frida Kahlo: arte y dolor (http://cvc.cervantes.es/aula/pasatiempos/ pasatiempos $2 /$ intermedio/gramatical/19111999_01.htm

c) ¡Uh, qué miedo! (http:/cvc.cervantes.es/aula/pasatiempos/ pasatiempos2/ intermedio/ gramatical/14012000_01.htm).

d) El diario de Laura (http:/cvc.cervantes.es/aula/pasatiempos/pasatiempos2/ intermedio/gramatical/19071999_01.htm\#juego).

e) Futuros diferentes (http://cvc.cervantes.es/aula/pasatiempos/pasatiempos2/ intermedio/gramatical/30052000_01.htm).

En Lecturas el profesor tiene que seleccionarlas para la práctica gramatical, ya que el objetivo de esta sección es desarrollar la destreza de comprensión lectora a través de textos que se acompañan de actividades sobre los contenidos lingüísticos y culturales que en ellos aparecen. Como en el caso anterior, destacamos las siguientes actividades

a) Nivel inicial: El sueño de Otto. Actividad 1: A los tres años (después de leer) (http:// cvc.cervantes.es/aula/lecturas/inicial/lectura_05/despues/actividad_01. htm). 
b) Nivel inicial: Gente que lee. Actividad 4: Gustos y preferencias (después de leer) http:/cvc.cervantes.es/aula/lecturas/inicial/lectura_04/dcspues/actividad_04.htm).

c) Nivel intermedio: Memorias de septiembre. Actividad 3: ¿Dije o deci? (después de leer) http://cve.cervantes.es/aula/lecturas/intermedio/lectura_10/despues/actividad_03.htm).

d) Nivel intermedio: Memorias de septiembre. Actividad 4: Ponlo en pasado (después de leer) (http:/cvc.cervantes.es/aula/lecturas/intermedio/lectura_10/ despues/actividad_04.htm).

e) Nivel avanzado: Do de pecho. Actividad 2: Una cuestión de forma (después de leer) (http:/cve.cervantes.es/aula/lecturas/avanzado/lectura_08/despues/actividad_02.htm)

f) Nivel avanzado: Los labios de Bárbara. Actividad 2: Como si lo supiera (después de leer) (http://cvc.cervantes.cs/aula/lecturas/avanzado/lectura_01/despues/actividad_02.htm).

3. Focalización para el desarRollo de COMPETENCIAS Y aCtividades orientadas a LA ACCIÓN

La lectura de este epígrafe hace que orientemos nuestra mirada hacia lo postulado en el Marco común europeo de referencia para las lenguas: enseñanza, aprendizaje y evaluación (MCER) y adoptemos los conceptos y orientaciones que en él se nos proponen.

Los alumnos utilizarán las ATL como recurso para llevar a cabo actividades de lengua ${ }^{8}$ y para ello activarán competencias generales ${ }^{9}$-en concreto, su capacidad para aprender --, y competencias comunicativas de la lengua ${ }^{10}$ - centradas sobre todo en el componente lingüístico-gramatical ${ }^{11}$, que repercutirá, lógicamente, en las pragmáticas--.. Concretando un poco más, podemos decir que la capacidad de aprender (saber aprender) se centraría en; a) las destrezas de estudio: la capacidad do utilizar materiales disponibles para el aprendizaje independiente y de organizar y utilizar materiales para el aprendizaje autodirigido; y, b) las destrezas heurísticas (de descubrimiento y análisis): la capacidad de utilizar nuevas tecnologías (por ejemplo, para buscar información en bases de datos, hipertextos, etc.). $\mathrm{Y}$, los lingüístico-gramaticales se centrarían en los parámetros y descriptores usados en la organización gramatical (elementos, categorías, clases, estructuras, procesos descriptivos y relaciones). Somos conscientes de que en este punto debemos acotar conceptos para no cacr en propuestas que cn la actualidad todavía son ulópicas desde el punto de vista tecnológico, nos estamos refiriendo al hecho de que con el uso de estas aplicaciones y recursos se po-

8 «Suponen el ejercicio de la competencia lingitística comunicativa dentro de un ámbito especifica a la hora de procesar (en forma de comprensión, cxpresión, intcracción y mediación) uno a más textos con el fin de realizar una tarea. (...) La competencia lingüística comunicativa que tiene el alumno o usuario de la lengua sc pone cn funcionamiento con la realización de distintas actividades de la lengua que comprenden la comprensión, la expresión, la interacción o la mediación (en concreto, interpretando o traduciendo). Cada uno de estos tipos de actividades se hace posible en relación con textos en forma oral o escrita, o en ambas» ( $M C E R$ ).

9 «No se relacionan directamente con la lengua, pero a las que se puede recurrir para accioncs de todo tipo, incluyendo las actividades de lingüísticas» (MCER).

10 «posibilitan a una persona actuar utilizando especilicamente medios lingüssticos) (...) Se puede considerar que la competencia comunicativa comprende varios componentes: el lingǘístico (competencia léxica, gramatical, semántica, fonológica, ortográfica y ortoépica), el sociolingüistico y el pragmático (discursivo, funcional y organizativo). Se asume que cada uno de estos componentes comprende, en concreto, conocimientos, destrezas y habilidades» (MCER).

11 «La compctencia gramatical se puede definir como el conocimiento de los recursos gramaticales de una lengua y la capacidad de utilizarlos» (MCER). 
drán trabajar conocimientos declarativos vinculados estrechamente con el uso del verbo y creemos que de forma autónoma y grupal. En opinión de F. Sanchez:

Con los medios técnicos de los que disponemos actualmente, la granática que se preste mejor a un tratamiento en un medio informatizado será aquella que presente reglas de funcionamiento que se puedan explicitar de manera sencilla, para las que existan normas de uso, y que puedan proporcionar un conocimiento declarativo. Todos hemos tenido la experiencia de encontrarnos con aspectos gramaticales para los que no se pueden generalizar una norma de uso $(2002: 413)$,

Lo dicho en el párrafo anterior no se pueda conseguir con éxito si no conocemos y fomentamos el uso de las distintas formas de aprender, o lo que es lo mismo de las estrategias de aprendizaje. Teniendo en cuenta que nucstra propucsta de ATL y recursos cstá dirigida, en su mayoría, a la práctica autónoma del alumno y a la mejora de sus competencias, creemos que son las estrategias directas cognitivas y las indirectas metacognitivas y afectivas las que utilizará para la consecución de su aprendizaje (Oxford, 1990, 1992/93).

\section{Momentos favorables EN El aUla para EL uso DE LAS ATL y OTROS RECURSOS EN RED}

Para integrar los recursos didácticos que hemos mencionado en la dinámica general de una clase, determinar su utilidad o no frente a otros recursos y ver si impulsa el proceso de enseñanza/aprendizaje del alumno, hay que analizar lo que Olga Juan ha denominado como «contextos de uso de la red»:

1. Obtener información y acceder a materiales reales. 2. Favorecer los intereses del individuo frente al grupo. 3. Procesar la información y trabajar con ella. 4. Adaptarse a las necesidades cognitivas del estudiante. 5. Fomentar los mecanismos de interacción y comunicación. 6. Completar lo trabajado en el aula. 7. Enriquecerse culturalmente. 8. Prestar mayor atención a las destrezas de producción (2001:21, 27-41).

Partiendo de esta taxonomía y teniendo en cuenta los objetivos que hay que alcanzar, los beneficios que aporta al estudiante, los requisitos que deben tenerse en cuenta cuando se hace uso de la red para la realización de una tarea y cl tipo de aplicaciones que son, creemos que las situación de aula que puede verse más favorecida con el uso de estas ATL en línea son:

a) Obtener información puntual sobre cualquier duda en relación con el uso de tiempos, personas, ... verbales; en este sentido, sería un material de consulta.

b) Completar lo trabajado en el aula, bien de forma presencial, semipresencial u on-line, ya que con ellas pueden practicarse estructuras que precisan de refuerzo por parte del alumno; su diseño está orientado hacia una consulta de tipo individual y complementan otras actividades de aula (Cruz Moya, 2002:451); aunque en el aula presencial, la consulta de los sitios web pueden realizarse perfectamente en parejas; en este punto juega un papel importante el espacio físico del entorno: ¿cuántos ordenadores hay en el aula?, ¿es necesario cambiar de aula para poder utilizarlos?, ¿es un espacio estático o dinámico?, etc., ya que de ello dependerá el logro de los objetivos que nos hayamos propuesto.

c) Apoyar el trabajo en el aula presencial. Este apartado estaría relacionado con el anterior, aunque aquí el uso de las aplicaciones y recursos sería en el aula; por lo tanto, habrá 
que valorar su utilidad teniendo cn cucnta el entorno físico. Cualquier método de los que hay disponibles en las librerías para la enseñanza/aprendizaje de E/LE incorporan actividades formales muy concretas, por cjemplo, rellenar formas verbales en una tabla/cuadro tras escuchar una audición o leer un texto, seleccionar entre varias formas verbales la adecuada para un contexto oracional y textual, completar oraciones y textos con la forma correcta de un verbo (que se suele ofrecer en infinitivo), subrayar las formas verbales de un texto, etc.; por lo tanto, las aplicaciones y recursos de los que nos venimos ocupando en este lugar podrían sustituir a las actividades en formato papel (aquí el profesor tendrá que controlar y valorar su uso, ya que en casi todos los ejercicios se ofrecen las soluciones), o servir de apoyo para su realización, por ejemplo, consulta a páginas con información gramatical para resolver un ejercicio concreto sobre el uso de los diferentes tiempos de pasado.

d) Material para que el alumno realice su autoevaluación. Por ejemplo, si un profesor de E/LE está utilizando para sus clases cualquier método de los que antes hemos comentado, sabrá que al final de cada unidad sucle haber una sección para que el alumno realice la autoevaluación sobre su proceso de aprendizaje. $\mathrm{Y}$, vistas algunas de ellas, es perfectamente posible sustituir determinadas actividades gramaticales en soporte papel por actividades en red, puesto que sus características son muy similares.

El término clave para poder utilizar estas aplicaciones en el contexto de ELE es considerarlos como posibilitadoras del proceso de aprendizaje (Sánchez, 2002:411), es decir, no debcn scr concebidos para un aprendizaje integral y autónomo, sino como complemento de una enseñanza presencial, semipresencial y on-line y en los tres casos deberá ser siempre guiada; de esta forma el profesor/tutor verá proyectadas algunas de sus funciones, por ejemplo, posibilitador del aprendizaje, guía, fuente de recursos, etc. (Instituto Cervantes, 1994:87-89), ya que la efectividad de estos recursos y aplicaciones va a depender, por su propio diseño, de la labor del tutor como guía de aprendizaje y gestor de la información, por lo que sería recomendable proporcionar una ayuda para su correcto uso, ya que algunos de los sitios web, que hemos mencionado en estas páginas, no la llevan en su diseño una descripción sobre su dinámica o funcionamiento, aunque la intuición y la lógica es lo que suele primar en cstos casos.

\section{5. ¿QUÉ PIENSA EL PROTAGONISTA DEL APRENDIZAJE SOBRE EL USO DE ESTOS RFCURSOS?}

Como escribió R. Sitman al comentar el uso de Internet en el aula: «los protagonistas son los estudiantes y en el trasfondo, los profesores que dirigen la función detrás de las bambalinas» (1998:5), por lo tanto, es fundamental, para poder realizar una evaluación sobre los recursos digitales ${ }^{12}$, saber cuál es la opinión que sobre cllos tiene el estudiante y que debe plasmar, por ejemplo, en una plantilla que contemple aspectos como los siguientes ${ }^{13}$ :

12 El profesor deberá evaluar el la integración de estos recursos en la dinámica general del aula; para ello recomendamos consultar las plantillas de valuación y comentarios de O. Juan Lázaro (2001:44-65, 67-68), J. Fcrnández Pinto y O. Juan Láraro (2000), M. Higueras García (2002), M. Cruz Piñol (2002), L. Codina (2000), P. Marquès (1999).

13 Como es lógico, según los recursos que utilice el alumno, se podrán o no rellenar todos los datos de la plantilla. 


\begin{tabular}{|c|c|}
\hline MI NOMBRE: & \\
\hline URL: http:// & \\
\hline Nombre del recurso/ejercicio/actividad & \\
\hline Autor/Institución: & \\
\hline Fecha (día que he utilizado el recurso): & 11 \\
\hline $\begin{array}{l}\text { ¿Qué navegador he utilizado?: } \\
\text { - Internet Explorer................... } \square \\
\text { - Netscape......................... } \square \\
\text { - Mozilla.......................... } \square \\
\text { - Opera............................ } \square\end{array}$ & 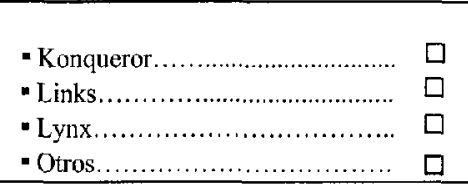 \\
\hline $\begin{array}{l}\text { ¿Qué recurso he usado?: } \\
\text { - Aplicación de ayuda a la escritura } \square \\
\text { - Conjugador verbal................. } \\
\text { - Flexionador verbal............... }\end{array}$ & $\begin{array}{l}\text { - Lematizador verbal.................. } \square \\
\text { - Información verbal................ } \square \\
\text { - Ejercicio de práctica verbal........ }\end{array}$ \\
\hline ¿Para qué he utilizado ese recurso?: & \\
\hline ¿He realizado algún ejercicio? ¿Cuánto & s? ¿Cuánto tiempo he tardado en realizarlo? \\
\hline $\begin{array}{l}\text { ¿Qué tipo de ejercicio es?: } \\
\text { - Rellenar huecos con palabras..... } \square \\
\text { - Relacionar elementos............ } \square \\
\text { - Seleccionar la opción correcta... } \square \\
\text { - Arrastrar la palabra correcta..... } \square \\
\text { - Pulsar/marcar con un clic......... } \square \\
\end{array}$ & $\begin{array}{l}\text { - Ordenar elementos........................ } \square \\
\text { - Hacer crucigramas.................. } \square \\
\text { - Respuesta abierta (enviar al tutor)... } \square \\
\text { - Otros................................... } \square\end{array}$ \\
\hline ¿Qué tjpo de ejercicios prefiero hacer? & \\
\hline $\begin{array}{l}\text { Prefiero utilizar estos recursos en: } \\
\text { - En el aula de forma individual... } \square \\
\text { - En el aula con mis compañeros.. } \square\end{array}$ & $\begin{array}{l}\text { - Fuera del aula de forma individual.. } \square \\
\text { - Otros................................ }\end{array}$ \\
\hline $\begin{array}{l}\text { Prefiero que la corrección sea: } \\
\text { - Automática y tcuándo?............ } \\
\text { - De acierto/error.............. } \\
\text { - Muestre una ayuda.......... } \\
\text { - Ofrezca pistás................ } \\
\text { - Inmediatamente............. } \\
\text { - Al final..................... }\end{array}$ & 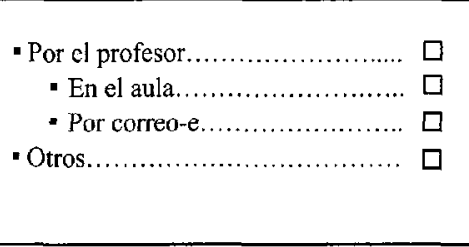 \\
\hline $\begin{array}{l}\text { ¿Las instrucciones/ayuda para usar este } \\
\text { - Si............................ } \square \\
\text { - Regular..................... } \square \\
\end{array}$ & $\begin{array}{l}\text { recurso son comprensibles y útiles? } \\
\text { - No ................................ } \\
\text { - No lleva ayuda/instrucciones...... } \\
\end{array}$ \\
\hline 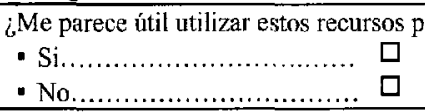 & $\begin{array}{l}\text { ara mi aprendizaje? } \\
\text { Para... }\end{array}$ \\
\hline ¿Qué he aprendido? & \\
\hline ¿Qué es lo que más me ha gustado? & \\
\hline ¿Qué es lo que menos me ha gustado? & \\
\hline ¿Me parece atractivo este lugar wcb? & \\
\hline $\begin{array}{l}\text { Prefiero utilizar estas aplicaciones } / \text { recur } \\
\text { - En formato papel............... } \square\end{array}$ & $\begin{array}{l}\text { sos: } \\
\text { - En formato digital (en red) ......... }\end{array}$ \\
\hline Comentarios / sugerencias: & \\
\hline
\end{tabular}




\section{Conclusiones}

La implementación de estas ATL y recursos a los programas de enseñanza-aparendizaje de $\mathrm{E} / \mathrm{LE}$ debe suponer un nuevo paso en este camino hacia la perfecta y deseable interactividad entre el aprendiz y los contenidos $\mathrm{c}$ interacción entre los protagonistas del aprendizaje, pero como decía en otro lugar de este trabajo: son muchos los cabos sueltos que todavía quedan por atar.

Desde el punto de vista que hemos adoptado debemos concluir, como docentes y por la propia naturaleza de las aplicaciones y recursos presentados, que tenemos que realizar su adaptación al contexto de aprendizaje del alumno, posibilitarle el desarrollo de estrategias de aprendizaje, guiarle hacia un conocimiento autorreflexivo y hacia el manejo adecuado de estos recursos didácticos, ya que su éxito dependerá de un saber hacer por parte del profesor/tutor. Pensemos que el uso de estas aplicaciones en línea debe ser motivador y, sobre todo, eficaz para el alumno y el profesor.

Estamos seguros de que los avances tecnológicos y la constante labor investigadora de los especialistas harán que dentro de poco ticmpo la integración de estos recursos en el proceso de enseñanza-aprendizaje de una lengua sea una realidad palpable y accesiblc potenticalmente a todos los protagonistas de esc proceso; sólo hace falta mirar hacia atrás y comprobar los avances de los últimos años.

\section{REFERENCIAS BIBLIOGRÁFICAS}

Arrarte, G. y J. Llisterri (1997). «Industrias de la lengua y enseñanza del español como lengua extranjera», Carabela, 42, Monográfico: Nuevas tecnologias y enseñanza del E/LE, págs. 27-38.

Arrarte, G. y J. I. Sánchez de Villapadierna (2001): Internet y la enseñanza del español. Madrid, Arco/Libros.

Arrarte, G. y J. I. Sánchez de Villapadierna (2002): «Lingu@nct Europa: un centro de recursos virtual y multilingüe para la enseñanza de lenguas». kn A. M. Gimeno Sanz (cd.): Actas del XII Congreso Internacional de ASELE. Tecnologias de la Información y de las Comunicaciones en la Enseñanza de ELE. Valencia, Universidad Politécnica de Valencia, págs. 327-333.

Barlo Ottonello, M. (2002): «Evaluación de la ejercitación gramatical en materiales didácticos multimedia: aspectos cognitivos, psicolingüísticos y didácticos\%. En A.M. Gimeno Sanz (ed.): Actas del XII Congreso Internacional de ASELE. Tecnologias de la Información y de las Comunicaciones en la Enseñanza de ELE. Valencia, Universidad Politécnica de Valencia, págs. 493-503.

Casanova, L. (1998): Internet para profesores de español. Madrid, Edelsa.

Codina, L. (2000): «Evaluación de recursos digitales en línea: conceptos, indicadores y métodos», Revista Española de Documentación Cientifica, 23/1, págs. 9-44.

Consejo de Europa (2002): Marco común europeo de referencia para las lenguas: enseñanza, aprendizaje y evaluación Madrid, Ministerio de Educación, Cultura y Deporle, Anaya. También en: http:/cvc.cervantes.cs/obref/marco/

Cruz Moya, O. (2002): «El aprovechamiento de Internet en E/LE: diseño y evaluación de materiales». En A. M. Gimeno Sanz (ed.): Actas del XII Congreso Internacional de ASELE. Tecnologias de la Información y de las Comunicaciones en la Enseñanza de ELE. Valencia, Universidad Politécnica de Valencia, págs. 449-458.

Cruz Piñol, M. (2002). Enseñar español en la era de Internet. Madrid, Octaedro. http://www.octaedro. com/prod_show.asp?art_no $=10041$

Cruz-Piñol, $\bar{M}$. (2004): «Presencia (y ausencia) de los hipermedios y de los géneros electrónicos en las Webs para la cnscñanza-aprendizaje del español como lengua extranjera (ELE)», Red ELE, 
1: http:/formespa.rediris.es/revista/cruz pinol.htm (12-04-2004). También en (2003): Cultura y educación, 15(3), págs. 311-325.

Diez Orzas, P. L., A. Martín de Santa Olalla y Í. Sánchez Paños (1997): «E/LE, Multimedia y tecnología lingüística: las cuatro destrezas», Carabela, 42, Monográfico: Nuevas tecnologias y enseñanza del E/LE, págs. 123-135.

Elder, D. (2001): «Para analizar un sitio Intemet», Tonos Digital. Revista Electrónica de Estudios Filológicos, 1: http://www.um.es/tonosdigital/znuml/estudios/elder $\mathrm{htm}$.

Fernández. Pinto, J. (2003-04): «Enseñar vocabulario en la red». Material del Curso de Experto Universitario en Nuevas Tecnologias en la enseñanza-aprendizaje del español como lengua extranjera. Universidad de la Rioja. Curso 2003-2004: http:/www.campusvirtual.unirioja.es/postgrados/espanol/index.html.

Fernández Pinto, J. y O. Juan Lázaro (2000): «Criterios de evaluación de materiales E/LE en red». Cuadernos Cervantes de la Lengua Española, 28 págs. 70-85: http://www.cuadernoscervantes. com/multi_28_criteeval.html

Gallego Arrufat, $\bar{M}^{\mathrm{a}}{ }^{\mathrm{J}}$ J. (1998): «Investigación en el uso de la informática en la enseñanza», Pixel-Bit. Revista de Medios y Educación, 11, págs. 7-31, http://www.sav.us.es/pixelbit/articulos/n11/n11art/ art111.htm

Grande Rodríguez, V. y F. J. Grande Alija (2002): «La gramática en la red: ¿qué pueden encontrar nuestros estudiantes? . En A. M. Gimeno Sanz (ed.): Actas del XII Congreso Internacional de ASELE. Tecnologías de la Información y de las Comunicaciones en la Enseñanza de ELE. Valencia, Universidad Politécnica de Valcncia, págs. 541-550.

Higueras García, M. (2002): «Criterios para la elaboración y selección de actividades comunicativas con Internets. En L. Miquel y N. Sans (eds.) Didáctica del español como lengua extranjera, 5 , págs. 109-121.

Instituto Cervantes (1994): La enseñanza del español como lengua extranjera. Plan Curricular del Instituio Cervantes. Publicaciones del Instituto Cervantes.

Juan Lázaro, O. (2001): La Red como material didáctico en la clase de E/LE. Madrid, Edelsa.

Llisterri, J. (2003a): «Lnseñanza del español y nuevas tcenologías», Curso La enseñanza del español como lengua extranjera, Departamont de Filologia Espanyola, Universitat Autònoma de Barcelona - Instituto Cervantes, Barcelona, 23 May 2003: http:/liceu.uab.es/ joaquim/applied_linguistics/ IC_UAB_03/IC_UAB_03.html.

Llisterri, J. (2003b): «Lingüística y tecnologías del lenguaje», Lynx. Panorámica de Estudios Lingüisticos, 2, págs. 9-71: http://liceu.uab.es/ joaquim/publicacions' TecnoLing_Lynx02.pdf.

Llisterri, J. (2003c): «Nuevas tecnologías y enseñanza del español como lengua extranjera», Curso $\mathrm{La}$ enseñanza del español como lengua extranjera III (Nivel Avanzado), Universidad Internacional Menéndez Pelayo, Santander, 25-26 August 2003: http://iceu.uab.cs/ joaquim/applied_linguistics/UIMP_03/UIMP_03.html.

Marquès, P. (1999): «Plantilla para la catalogación, evaluación y uso contextualizado de páginas web»: http://dewey.uab.es/pmarques/evalwcb.htm.

Marti Antonín, $M^{2}$. A. y J. Llisterri (2001): «La ingeniería lingüistica en la sociedad de la información», Digit·HVM Revista digital d'humanitats, 3: http:/www.uoc.edu/ humfil/articles/esp/listerri-marti/llisterri-marti.html.

Martín Mohedano, $M^{\natural}$ (2003): «La enseñanza del léxico español a través de Internet», Frecuencia. Revista de Didáctica Español Lengua Extranjera, 24, págs. 40-43.

Oxford, R. (1990): Language Learning Strategies: What Every Teacher should Know. New York, Newbury House.

Oxford, R. (1992/93): «Language Learning Strategics: Update and ESL suggestions», TESOI Journal, 2(2).

Sánchez Paniagua, F. (2002): «Proyecto de un dispositivo informático para un aprendizaje de la gramática de manera autónoma. Español, nivel intermediò. En A.M. Gimeno Sanz (ed.): Actas del 
XII Congreso Internacional de ASELE. Tecnologias de la Información y de las Comunicaciones en la Enseñanza de ELE. Valencia, Universidad Politécnica de Valencia, págs. 411-419.

Sitman, R. (1998): «Divagaciones de una internauta. Algunas reflexiones sobre cl uso y abuso de la Internet en la enseñanza del ELE». Boletín de ASEIE, 12, págs. 7-33. También en Espéculo, 10: http://www.ucm.es/info/especulo/numerol0/sitman.html

Vera Luján, A. (1994): Fundamentos de análisis sintáctico (de la palabra al texto). Murcia, Universidad de Murcia, Secretariado de Publicaciones. 